\title{
The Recognition of Basal Ganglia Area in the Brain MRI Image with Hybrid Classifier
}

\author{
Min-Chi Wu, Chiun-Li Chin, Wen-Chi Chin, Jian-Shiun Wu, Wan-Jou Li, and Ting-Yu Liu
}

\begin{abstract}
To understand the relationship between the volume of basal ganglia and parkinson's disease, this paper proposes the recognition of basal ganglia in the brain MRI image with hybrid classifier. It can locate the position of basal ganglia in the 2D brain MRI image. It employs three feature extraction methods to extract the texture feature of basal ganglia. The three feature extraction methods include GLCM, Law's mask and Hu's method .Next, we uses rank search method and genetic search method to perform feature selection. It can reduce computation complexity. Finally, hybrid classifier combining neuro-fuzzy system and adaboost algorithm is used to achieve recognition. From experimental result, we discovered that our proposed method has the high recognition rate.
\end{abstract}

Index Terms-Parkinson's disease, magnetic resonance imaging, basal ganglia, feature pooling, neural-fuzzy-based adaboost.

\section{INTRODUCTION}

The motive of automatic medical image segmentation is to describe the image content based on its features. The segmentation of brain images can provide useful information about neuro-generative diseases. MR image segmentation is fundamental in obtaining anatomical and functional information from biological structures and tissues, and it is used towards visualization, surgical guidance, quantitative analysis, and diagnosis. Manual segmentation of brain images is a tedious task however, which is why a variety of methods have been developed for automated segmentation. Manual segmentation is tedious, time consuming, and often not reproducible. For a variety of tissues and organs function and metabolism disorder diagnosis ability, become the biggest characteristic of MRI.

The volume of basal ganglia in brain medical image analysis, it has been the focus of investigation in clinical studies of Parkinson disease. Traditional Parkinson's disease needs a doctor long-term observation of the patient's symptoms and has a way to judge whether they have Parkinson's. For studying the volume change of basal ganglia, its segmentation in a MRI image is the first processing step. Currently, MRI technology has been widely used in various medical diagnostic. However, not all MRI images are so clear and easy to interpret, many small lesions characteristic

Manuscript received October 9, 2016; revised January 12, 2017.

Wen-Chi Chin, Jian-Shiun Wu, Wan-Jou Li, Ting-Yu Liu and Chiun-Li Chin are with the Department of Medical Informatics, Chung Shan Medical University, Taichung, Taiwan (e-mail: nemocandy5@gmail.com, lover0258020@gmail.com, joy5960829@gmail.com, ernestli@csmu.edu.tw).

Ming-Chi Wu is with the Department of Medical Imaging, Chung Shan Medical University Hospital, Taichung, Taiwan (e-mail: hao620620@gmail.com). cannot be determined in accordance with the naked eye. Noise and resolution are the two main factors that affect image quality, low noise and high resolution images which ideal used in medical diagnostics. In the past literature, Wang et al. [1] proposed a simple but generally applicable approach to improving the accuracy of automatic image segmentation algorithms relative to manual segmentations. In this paper, the wrapper method attempts to learn the intensity, spatial and contextual patterns associated with systematic segmentation errors produced by the host method on training data for which manual segmentations are available. Then, it attempts to correct such errors in segmentations produced by the host method on new images. Finally, this paper has high segmentation success rate. Yousef [2] proposed a new algorithm to split the spinal fluid in the brain's gray matter and white matter. This method mainly divided into two parts. The first part uses Active Contour Model (ACM) to segment the region of the brain. The second part based on binary mathematical morphology and region growing method framework selected target organizations. Markov Random Field (MRF) [3] have a good effect on the image noise removal but MRF has high time complexity, so they raised an algorism based on Markov Random Field (MRF) and Ant Colony Optimization (ACO) to achieve faster convergence to improve execution speed of the system. Several experiments on phantom and real images were performed. Results indicate that the proposed algorithm outperforms the traditional MRF and hybrid of MRF-ACO in speed and accuracy. This paper [3] proposed a different approach for the segmentation of neonatal brain MRI, based on the infusion of high-level brain morphology knowledge, regarding relative tissue location, connectivity and structure. Their method does not require manual interaction, or the use of an atlas, and the generality of its priors makes it applicable to different neonatal populations, while avoiding atlas-related bias. Because MRI images [4] have more or less uneven brightness condition, they improved Kernel-Based Fuzzy c-Means to give it a better noise performance and higher segmentation accuracy. They also proposed a new method to determine the starting position of automation, this method effectively overcome the noise and prevents blurred edges. Methods for fully automatic brain tissue classification typically rely on an existing anatomical model for localizing a training set for each tissue class to be labelled, e.g. gray matter, white matter, and cerebro-spinal fluid (CSF). Knowledge of the exact spatial distribution of brain tissues in images acquired by MRI is necessary to measure and compare the performance of segmentation algorithms [5]. It is to use genetic algorithm [6] combined with K-means classification algorithms to separate MRI images and convert them into 3D images allowing surgeons to perform preoperative planning, but the time is 
too long execution system, and not suitable for acute brain surgery. Wu proposed [7] using Cubic Curve for contrast-enhanced images and using texture feature and contrast feature to achieve breast cancer detection. Many researchers proposed different feature extraction method to describe different medical image characteristics. The method of general feature extraction can be divided into two classes: texture based features [8]-[12] and intensity based statistical features [13]-[17].

From the above literature survey shows that intensity based statistical features are the straightest forward and have been widely used, but due to the complexity of the pathology in human brain and the high quality required by clinical diagnosis, only intensity features cannot achieve acceptable result. I such applications, segmentation based on textural feature methods gives more reliable results. Therefore, this paper will use multi-texture feature extraction method, including GLCM, Law's Mask and Hu's texture feature extraction method, to extract the feature of basal ganglia. And, the neural-fuzzy based Adaboost algorithm will be our classifier to train and test the extracted features.

The rest of the paper is organized as follows. Section II presents the preprocessing, feature extraction method, and our proposed hybrid classifier. In Section III the results are discussed and conclusion is presented in Section IV.

\section{MATERIAL AND METHOD}

As shown in Fig. 1, is a flow chart of the basal ganglia detect is divided into three parts, the first part is pretreatment, in this part of the brain in order to get the position, so we will get the image contrast enhancement, image binarization and noise processing to obtain the maximum circumscribed rectangle. The second part, in order to find the candidate regions of the basal ganglia area, we will, through image processing to determine whether there after the cerebrospinal fluid, so that we can find the candidate regions. The third part is to find the basal ganglia area, in order to pinpoint the location of the basal ganglia, we will use three different feature extraction methods are GLCM, Law's mask, Hu's feature extraction and feature values do training and testing, and finally get the base nuclear position. Then we will explain the steps of each process in detail.

\section{A. Preprocessing and Finding the Candidate Region}

We use brain MRI image to achieve this task. The image resolution is $208 \times 256$ and its format is DICOM. This step is to obtain clear image and find the candidate region of basal ganglia. The series of image processing method are used, including contrast enhancement, binary image processing and de-noise, etc. The original brain MRI image is shown in Fig. 2.

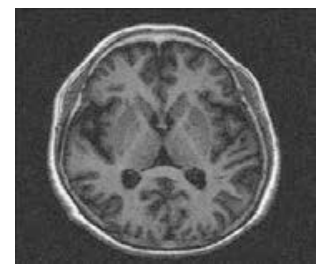

Fig. 2. Original image.
In contrast enhancement part, we use the cubic curve contrast enhancement method [8] to enhance the image contrast. This method uses a suitable inflection point to construct cubic curve to compensate the contrast of image.

Fig. 3 shows an enhanced brain image after performing cubic curve contrast enhancement.

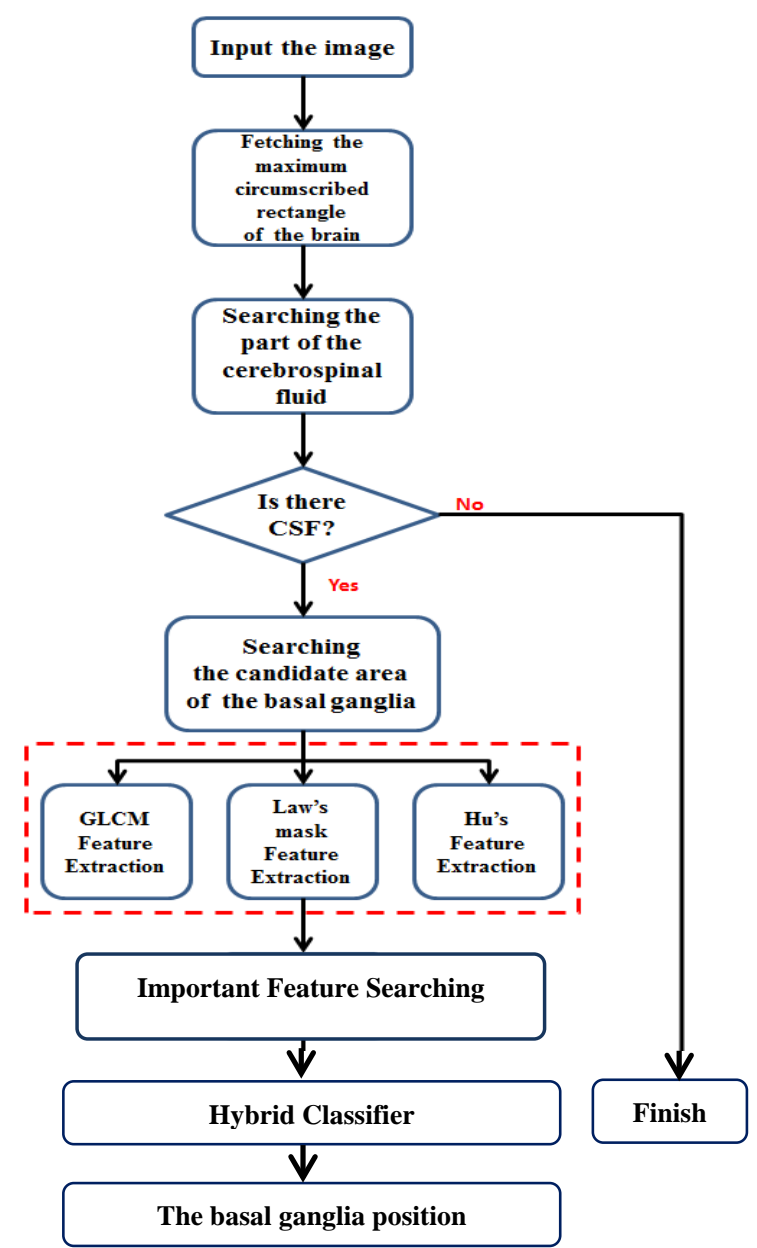

Fig. 1. The processing flowchart of basal ganglia area detection.

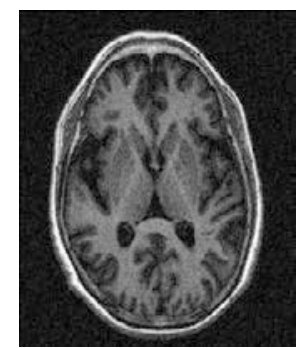

Fig. 3. Brain imaging after contrast enhancement.

Subsequently, continuation contrast image binarization part of our enhanced by the method of the optimal threshold of Otsu, proposed to binary image, the results are shown in 6 (a) of the brain of binary images. In part to the noise we use two ways to complete imaging technology in theoretical patterns of opening and closing of the way and manner median filter.

After the above, via brain imaging contrast enhancement, and then we will judge whether the cerebrospinal fluid image, the image will have its image long cerebrospinal fluid, the width at the center of each $1 / 3$ whichever candidate regions 
as the basal ganglia. As shown in Fig. 4.

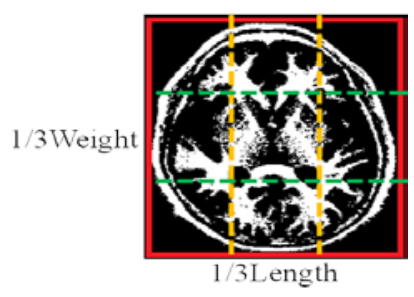

Fig. 4. One-third of candidates area is the basal ganglia.

Fig. 5 shows the result removed CSF image. Next, the basal ganglia area will be recognized from non-CSF area with $\mathrm{n} \times \mathrm{n}$ sliding window as show in Fig. 5. The sliding window will be used to extract feature. And, these features will be input to a hybrid classifier with learning ability. Finally, the sliding window area will be recognized whether it is basal ganglia area or not.

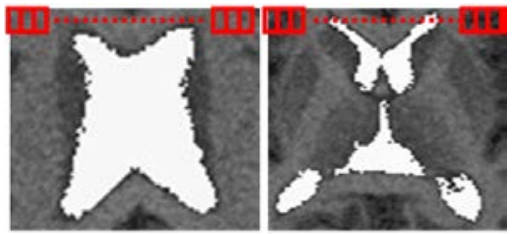

Fig. 5. One-third of candidates area is the basal ganglia.

Fig. 6 shows the image of basal ganglia area and non-basal ganglia area. The first row in the Fig. 6 shows basal ganglia image. The second row shows non-basal ganglia image. These images have the texture and intensity difference. Hence, in the next section, we will extract these features using different feature extraction method.

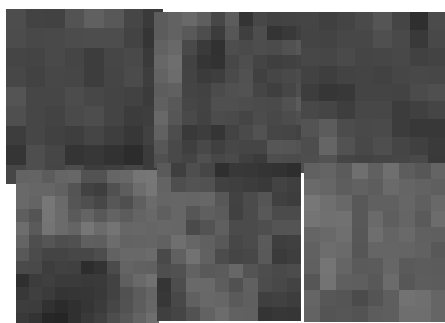

Fig. 6. One-third of candidates area is the basal ganglia.

\section{B. Feature Extraction}

After the above-mentioned process, we will use the basal ganglia to feature extraction. Image texture can be seen as an image area containing repeated patterns of pixel intensities arranged in some structural way. And after obtaining the candidate regions of the brain nuclear imaging substrate, we will respectively adopt GLCM, texture and Hu's Law's Mask to retrieve extracting feature vectors.

\section{B.1 First-order feature}

First-Order statistics feature measure the properties of an individual pixel values. They are not texture descriptor because they carry no information about comparative position of pixels with respect to one another, and measure the chance of observing gray value intensities at randomly chosen location in the given input image. Herein, we extract average intensity and standard deviation of image.

\section{B.2 Second-order feature- GLCM}

Commonly, MRI images appear in grey-level form.
However, the tonal intensity is not sufficient to describe each image texture class. The extraction of textural features is very important in the process of image segmentation. The most common class of textural features is based on the grey-level co-occurrence matrix (GLCM). And it is a common feature which describes the texture by studying the spatial correlation properties of grayscale. We extract the following five features: contrast, correlation, energy value, homogeneity and entropy. The formula is shown as follows:

(a). contrast

$$
\sum_{i, j}|\mathbf{i}-\mathbf{j}|^{2} p(i, j)
$$

(b). correlation

$$
\sum_{i, j} \frac{\left(i-\mu_{i}\right)\left(j-\mu_{j}\right) p(i, j)}{\sigma_{i} \sigma_{j}}
$$

(c). energy value

$$
\sum_{i, j} p(i, j)^{2}
$$

(d). homogeneity

$$
\sum_{i, j} \frac{p(i, j)}{1+|i-j|}
$$

(e). entropy

$$
\sum_{i, j} p(i, j) \log p(i, j)
$$

where $\mu$ represents mean and $\sigma$ represents variance. And $p$ $(i, j)$ corresponds to the elements of co-occurrence matrix, i.e. the probability of moving from a pixel with gray level $i$ to a pixel with gray level $j$.

\section{B.3 Law’s Mask}

First, we will be divided into a lot of small area which are $11 x 11$ of size for the basal ganglia brain syndrome candidate region images. If we find the black part, we will get rid of these parts and directly determine the air. We know that most of the black areas as the air, so we only for the candidate region in order to reduce the computing time.

Laws developed five labeled vectors which could be combined to form two dimensional convolution kernels. When convolved with a textured image these masks extract individual structural components of the image $[x]$. The five vectors are: $\mathrm{L} 5=[1,4,6,4,1], \mathrm{E} 5=[-1,-2,0,2,1]$, S5 $=[-1$, $0,2,0,-1], \mathrm{R} 5=[1,-4,6,-4,1]$ and W5 $=[-1,2,0,-2,1]$. Then these five masked interaction into 25 two-dimensional Laws' Masks to obtain 121 different feature points which size of 11x11. And then we'll take out TR of Laws' Mask calculated values and determine the texture. After a series of particular convolution with selected Laws' masks, the outputs are passed to texture energy measurement (TEM) filters for the analysis of the texture property of each pixel. These consisted of a moving nonlinear window operation; every pixel of the image is replaced by comparing the pixel with its local neighborhood based on five statistical descriptors (mean, standard deviation, Skewness, Kurtosis, and Entropy). Then, 
we obtain the value $\mathrm{TR}_{\mathrm{ij}}$ which represents Laws' Mask to calculate the following five features: Mean, SD, Skewness, Kurtosis, and Entropy. They are calculated as (6) to (10):

$$
\begin{gathered}
\text { Mean }=\frac{\sum_{i=0}^{M} \sum_{j=0}^{N}\left[T R_{i j}\right]}{M \times N} \\
\mathrm{SD}=\sqrt{\frac{\sum_{i=0}^{M} \sum_{j=0}^{N}\left(\mathrm{TR}_{\mathrm{ij}}-\mu\right)^{2}}{M \times N}} \\
\text { Skewness }=\frac{\sum_{i=0}^{M} \sum_{j=0}^{N}\left(T R_{i j}-\mu\right)^{3}}{M \times N \times \sigma^{3}} \\
\text { Kurtosis }=\frac{\sum_{i=0}^{M} \sum_{j=0}^{N}\left(T R_{i j}-\mu\right)^{4}}{M \times N \times \sigma^{3}}-3 \\
\text { Mean }=\frac{\sum_{i=0}^{M} \sum_{j=0}^{N}\left(T R_{i j}\right)^{2}}{M \times N}
\end{gathered}
$$

In this step, we will get 42 features from every little area, and then we will take advantage of these features to identify whether these areas of the brain basal ganglia.

\section{B.4 Hu's features}

Hu's is last method used in feature of extraction. It was derived from the theory of algebraic invariant. This technique is chosen to extract image features which generated features are rotation-scale-translation invariant. Moment invariants that are computed from each of the window are used to form feature vectors. A image of two qualitative $b(x, y)$ region's $(p+q)$ step space carves define a

$$
m_{p q}=\sum_{x} \sum_{y} x^{p} y^{q} b(x, y)
$$

where $x$ and $y$ are the horizontal and vertical image of position, If the probability density function's zero-order moment is contained within the area of the number pixels. The same can be defined as central moment:

$$
\begin{gathered}
\mu_{p q}=\sum_{x} \sum_{y}\left(x-x_{c}\right)^{p}\left(y-y_{c}\right)^{q} b(x, y) \\
x_{c}=\frac{m_{10}}{m_{00}} \text {, and } y_{c}=\frac{m_{01}}{m_{00}}
\end{gathered}
$$

The moments are further normalised for the effects of change of scale using the following formula:

$$
\eta_{p q}=\frac{\mu_{p q}}{\mu_{00}^{\gamma}}
$$

where the normalization factor: $\gamma=(p+q / 2)+1$. Hu's put forward a continuous function Square defined moments in 1962, and derivation. It has translation invariance, rotational invariance and proportion of invariance. The normalization in the second moment and third moment exporting seven invariant moments [14]. For different medical images, its invariant moments are usually significantly different, but after rotation, zoom in or out, feature vectors broadly similar. In this paper, the application of this feature is the based on identify position of the basal ganglia of the brain.

Subsequently, feature selection determines the effectiveness of identification. This paper proposes the way of combining two feature selection methods. The advantage is that can be more recognition of the feature selected. On the other hand, it can reduce a lot of loading caused by operation of the classifier. And also can reduce most of the time in the classifier training stage and let classifier ability identify further improved.

The feature selection method which we proposed is combined RankSearch [18] with GeneticSearch [19]. RankSearch is feature selection method, and it is achieved by cross-validating the attribute rankings with respect to a classification learner to find the best attributes while another is GeneticSearch. GeneticSearch is through the features of genetic algorithm to select the best combination.

The method to combine RankSearch and GeneticSearch is shown as the follow. First, all the samples of the dataset are inputted to GeneticSearch and we will obtain the best feature combination from GeneticSearch. Then, we determine the number of the best feature combination. If the number of the features is too many, the selected features will be inputted to RankSearch to be sorted by pros and cons, and then the worse features will be eliminated. On the other hand, if the number of the features is too few, we will input the features which are not selected to RankSearch and bring the better features into the selected features to accomplish the process of feature selection. Fig. 5 shows the flowchart of feature selection.

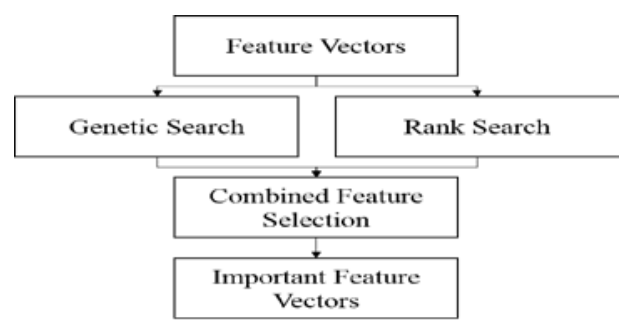

Fig. 7. The flowchart of feature selection.

It is effective to reduce the loading of training the classifier by using the combination of two feature selection method and the recognition ability of the features which we obtain are higher. In addition, it can reduce lengthy training time when we execute the classifier because of removing useless features and let the classifier more simplified. Therefore, we can get the training classifier model architecture faster and the recognition result can be more complete and accurate.

\section{Hybrid Classifier}

First, to model the target system, a training dataset of input/output pair $\left(x_{1}, x_{2}, x_{3}, \ldots, x_{n}, y\right)$ is required, and the sampling weights are initialized. The instance weights in the dataset are assigned uniformly. Subsequently, we use a neuro-fuzzy system to be a weak learner in Adaboost classifier. Neuro-fuzzy has been combined into ensembles to improve accuracy and robustness. Ensemble of classifiers is created when trained. The neuro-fuzzy system we used adopts SONFIN architecture. SONFIN is a method for tuning an existing rule base with a learning algorithm based on a 
collection of training data. It is supervised learning. Hence, the input/output are mapped adaptively by SONFIN through membership functions, rules, precise, and consequent parameters. SONFIN is trained to obtain hypothesis $h_{t}$. The

SONFIN can be used for normal operation at any time during the learning process without repeated training on the input-output patterns when on-line operation is required. There are no rules in the SONFIN initially. The training process continues till the desired least mean square is achieved. Second, the error in Eq. (15) is calculated by statistics of different label between the output of SONFIN and desired output.

$$
\varepsilon_{t}^{f}=\sum_{i=1}^{n}\left[d_{t}^{L}(i) \times\left(h_{t}^{f}\left(x_{i}\right) \neq y_{i}^{L}\right)\right]
$$

where $L$ is the number of classes. According to the number of classes $L$, the overall operation is be performed $L$ times continuously and the main function is to set the value which corresponded to the class to 1 and others to -1 . Namely, multi-class is transformed into two-class. Obviously, the error must never become zero. Therefore, before calculating Eq. (15), we constrain the error to be strictly positive. Herein, the $d_{t}(i)$ will be updated in step e in the algorithm of Fig. 3.

Then, the representative weak learner is selected. The weak learner has minima error value calculated from above step. When getting the representative weak learner, the weighting $\alpha_{t}$ will be calculated using Eq. (16). The formula is shown as follow. The final strong classifier will use the weighting value.

$$
\alpha_{t}^{L}=0.5 \times \log \left(\frac{1-\varepsilon_{t}^{L}}{\varepsilon_{t}^{L}}\right)
$$

The $\alpha_{t}$ can be interpreted as a weight showing the importance of the rule: unreliable, low performing rules have a reduced significance in the final classification decision, as compared to rules exhibiting a small error. The Eq. (17) is the update formula of $d_{t}$. Because the instance weights in the dataset are assigned uniformly, the distribution of the samples as input to the supervised base classifier is given in Eq. (15), where ' $n$ ' is the total number of instances in the data subset and $d_{t}$ is the distribution of the classifier ' $t$ '. It is divided into two parts. First, if the output of SONFIN is not equal to desired output (i.e. $\left.h_{t}\left(x_{i}\right) \neq y_{i}\right)$, the next $d_{t}$ will be updated using Eq. (17) (i.e. $\left.d_{t+1}(i)=d_{t}(i) \times \alpha_{t}\right)$. On the contrary, the $d_{t}$ will not be updated. Subsequently, it will be normalized in the Eq. (16).

$$
\begin{gathered}
d_{t}(i)=\frac{1}{n} \\
\text { if } \quad h_{t}\left(x_{i}\right) \neq y_{i}, \quad i=1 \ldots n \\
d_{t+1}(i)=d_{t}(i) \times \alpha_{t} \\
\text { else } \quad d_{t+1}(i)=d_{t}(i) \\
d_{t+1}(i)=\frac{d_{t+1}(i)}{\sum_{i=1}^{n} d_{t+1}(i)}
\end{gathered}
$$

The updated distribution samples are given as input for the next iteration. Similarly, the process is repeated for $\mathrm{T}$ iterations. The Adaboost algorithm repeatedly invokes a neuro-fuzzy system (SONFIN) on various distributions of the training data and to aggregate the individual classifiers into a single overall classifier. The distribution of training instances is changed based on the error the current classifier exhibits on the training set after each iteration. Adaboost combines the individual classifiers (fuzzy rules) considering their errors at the time of their generation. When an unseen instance is input in the classifier, the classification decision is made following a weighted maximum scheme:

$$
\begin{gathered}
H(x)=\arg \max \left(H_{L}\right) \\
\text { where } \quad H_{L}=\arg \max \left\{\sum_{t=1}^{T} \alpha_{t}^{L} h_{t}^{L}\left(x_{i}\right)\right\}
\end{gathered}
$$

The proposed Hybrid classifier algorithm is as shown in Fig. 6. The training distribution is updated by including the cost of misclassification on successive iterations. The updated distribution samples are given as input for the next iteration. Similarly, the process is repeated for $\mathrm{T}$ iterations. The instances can be classified into multi-class, namely, not only two classes. If the instances are classified correctly, the weight of these instances is reduced aggressively such that these instances will not be considered during next iteration of training. The training process continues till the desired least mean square is achieved.

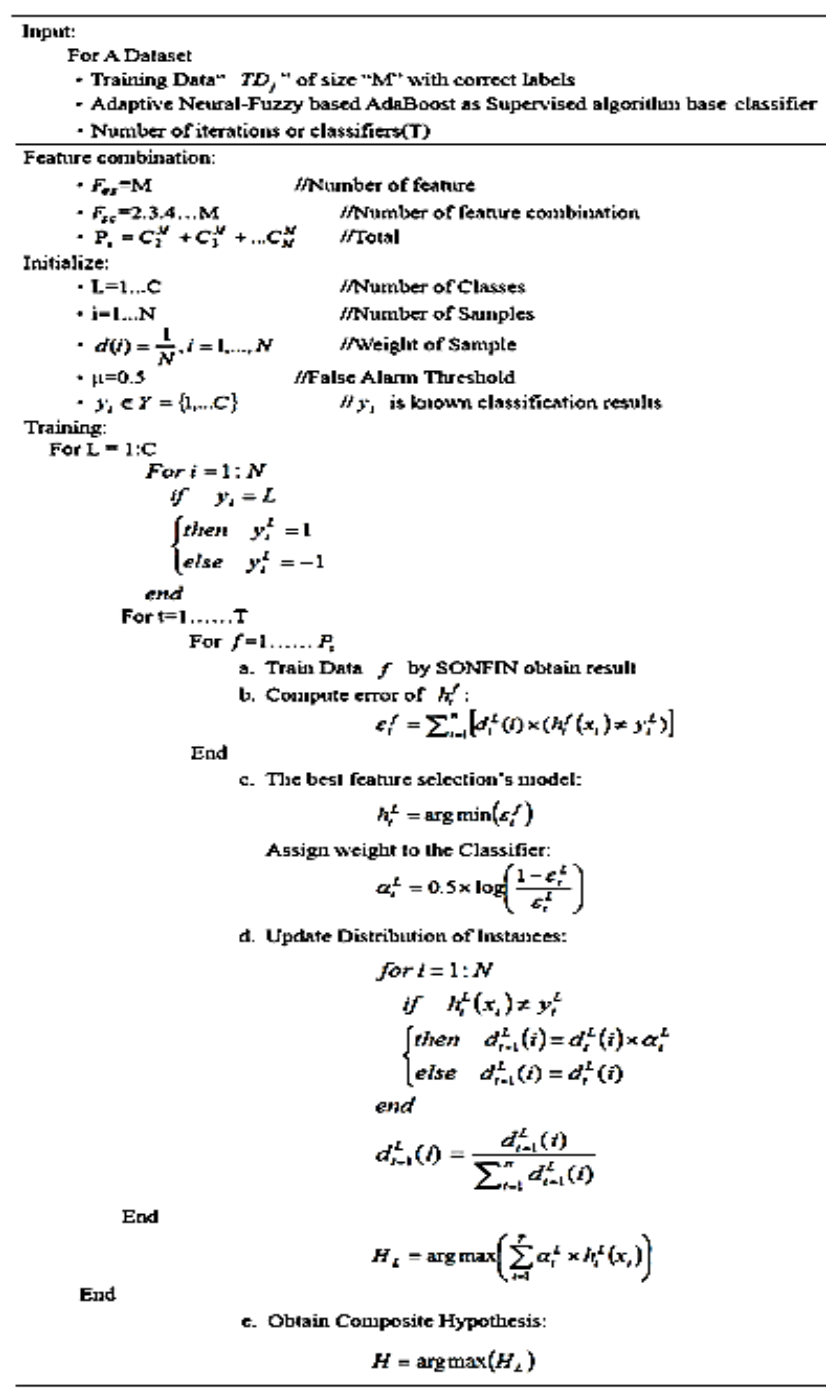

Fig. 8. Hybrid classifier algorithm. 


\section{RESULTS}

To validate the proposed method, we relied on the Chung Shan Medical University Hospital dataset within 2014. This dataset has 20 patients.

The images used in this paper are 1.5T MRI images in DICOM format with the size of $208 \times 256$ and the slice thickness of $1.0 \mathrm{~mm}$. Fig. 7 shows the partial original image. And, Fig. 8 shows the processed result image of our proposed method. The red area is basal ganglia area. For calculating our system successful recognition rate, radiologist helps us to manually select the basal ganglia area in the original image. These images are regarded as ground-truth image.
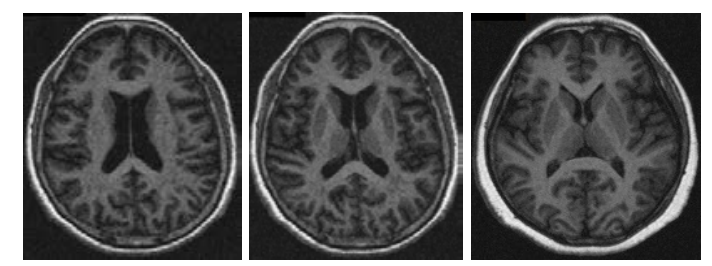

Fig. 9. The original image of brain 1.5T MRI.

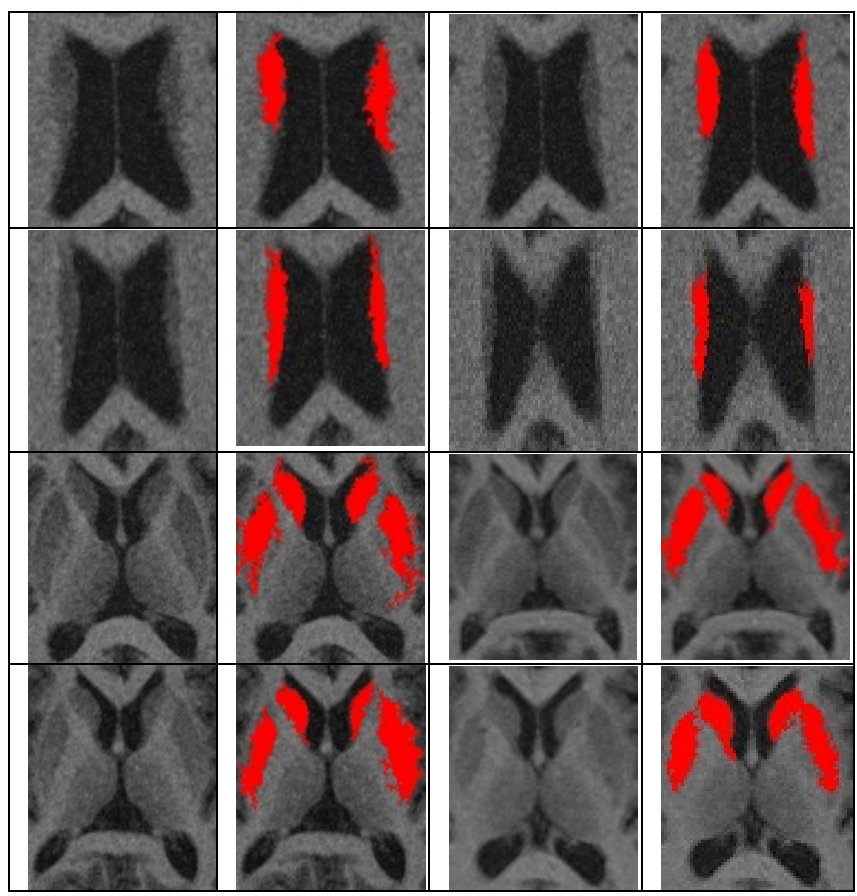

Fig. 10. The results of our proposed method.

Table I shows the comparison result among our proposed method and other method, including SONFIN, Adaboost, and SVM. Due to hybrid classifier integrates SONFIN and Adaboost, we compare these two methods. And, SVM classifiers are often used in classification. Hence, we use libsvm to perform testing with our proposed method.

TABLE I: THE COMPARISON RESUlt AMONG OUR PROPOSED METHOD AND OTHER METHOD

\begin{tabular}{cc}
\hline \hline Approach & $\begin{array}{c}\text { The correct } \\
\text { classification } \\
\text { percentage (\%) }\end{array}$ \\
\hline Our feature Selection +Hybrid Classifier & $92.3 \%$ \\
Our feature Selection +SONFIN & $92.0 \%$ \\
Our feature Selection +Adaboost & $91.3 \%$ \\
Our feature Selection +SVM(libsvm) & $91.1 \%$ \\
\hline \hline
\end{tabular}

Our purpose in this experiment was to test the computational cost of this approach. The experiment was run on a personal computer with Intel(R) Core(TM) i5-4200H CPU $2.8 \mathrm{GHz} \times 2$, $8 \mathrm{~GB}$ memory, and the operation system is Microsoft Win10. Table II shows the comparative time in terms classification with our proposed feature selection.

TABLE II: THE COMPARATIVE TIME IN TERMS CLASSIFICATION WITH OUR PROPOSED FEATURE SELECTION Approach Average time (min)

$\begin{array}{cc}\text { Our feature Selection +Hybrid Classifier } & 3.5 \\ & \\ \text { Our feature Selection +SONFIN } & 3.0 \\ \text { Our feature Selection +Adaboost } & 2.5 \\ \text { Our feature Selection +SVM(libsvm) } & 2.4\end{array}$

In addition, due to the popularity of X-ray / MRI imaging, doctors have more burden in examining images. To assist the doctor to detect images, we focused on the basal ganglia brain imaging features and made an auxiliary tool to identify the position of basal ganglia. It can accurately classify each image in the exact location of the basal ganglia. To reduce the burden of identification on physician, it supplied the output to the doctor for identification diagnosis data. We integrated and built an auxiliary tool to identify the position of basal ganglia of the brain. It fully automated help the doctor make the correct diagnosis and treatment planning.

\section{DisCUSSIONS AND CONCLUSIONS}

Currently the academic researches about detecting the basal ganglia are very limited, and it is possible to correctly detect the basal ganglia of the success rate is not high. The results are not necessarily accurate. We developed a system to detect the basal ganglia by image and pattern recognition technology. It can help doctors to diagnose and can effectively save time radiologist-diagnosed. From experimental result, we discovered that texture feature is useful for recognize basal ganglia. We also compared with other classifiers. The results are better than with SONFIN, Adaboost and SVM classifier. The results presented in this paper that the proposed method has $92.3 \%$ correct classification success rate. Our proposed method has a higher success rate than the other three methods; the reason is that we use a hybrid classifier, combining the advantages of SONFIN and Adaboost. Practical implementation requires careful consideration of the power of the individual features to discriminate between textures. Hence, our proposed feature selection is used before performing the classification and recognition. This is essential to reduce the influence that heavily correlated features, and features with little discriminatory power, have on the overall classification. Then we perform test in time consumption. From testing results, we understand that our proposed system takes a lot of time to complete, spend more than the other three methods time. In the future, the deep learning algorithm can be used to solve high time consumption problem.

\section{ACKNOWLEDGMENT}

This work was supported by research grants to Chung 
Shan Medical University Hospital (CSH-2013-A-023).

\section{REFERENCES}

[1] H. Z. Wang, R. D. Sandhitsu, J. W. Suh et al., "A learning-based wrapper method to correct systematic errors in automatic image segmentation: Consistently improved performance in hippocampus,cortex and brain segmentation," NeuroImage, vol. 55, pp. 968-985, 2011.

[2] S. Yousefi, R. Azmi, and M. Zahedi, "Brain tissue segmentation in MR images based on a hybrid of MRF and social algorithms," Medical Image Analysis, vol. 16, pp. 840-848, 2012.

[3] L. Gui, R. Lisowski, T. Faundez et al, "Morphology- driven automatic segmentation of MR images of the neonatal brain,” Medical Image Analysis, vol. 16, pp. 1565-1579, 2012.

[4] Z. X. Ji, Q. S. Sun, and D. S. Xia, "A framework with modified fast FCM for brain MR images segmentation,” Pattern Recognition, vol. 44, pp. 999-1013, 2011.

[5] B. Alfano, M. Comerci, M. Larobina et al, "An MRI digital brain phantom for validation of segmentation methods," Medical Image Analysis, vol. 15, pp. 329-339, 2011.

[6] G. M. Xian, "An identification method of malignant and benign liver tumors from ultrasonography based on GLCM texture features and fuzzy SVM," Expert Systems with Applications, vol. 37, pp. 6737-6741, 2010.

[7] S. Y. Wu, C. L. Chin, Y. S. Cho, Y. C. Chang, and L. P. Hsu, "Intelligent breast tumor detection system with texture and contrast features," accepted by Biomedical Engineering: Applications, Basis and Communications, 2012.

[8] O. Xiang, P. Wei, and P. Xiao, "In vivo skin capacitive imaging analysis by using grey level co-occurrence matrix (GLCM)," International Journal of Pharmaceutics, vol. 460, pp. 28-32, 2014.

[9] Q. G. Wu and Y. Gan, "An active contour model based on fused texture features for image segmentation,” Neurocomputing, vol. 151, pp. 1133-1141, 2015.

[10] U. R. Acharya, S. V. Sree, and M. M. R. Krishnan, "Atherosclerotic risk stratification strategy for carotid arteries using texture-based Features," ultrasound in medicine \& biology, vol. 38, pp. 899-915, 2012.

[11] T. T. Tang and W. H. Wang, "Vehicle classification for diverse camera viewpoints and scenes,” ICL Technical Journal, June $25^{\text {th }}, 2008$.

[12] A. B. Oktay, N. B. Albayrakb, and Y. S. Akgul, "Computer aided diagnosis of degenerative intervertebral disc diseases from lumbar MR images," Computerized Medical Imaging and Graphics, vol. 38, pp. 613-619, 2014.

[13] M. Vasantha, D. R. V. S. Bharathi, and R. Dhamodharan, "Medical image feature, extraction, selection, and classification," International Journal of Engineering Science and Technology, vol. 2, pp. 2071-2076, 2010.

[14] Y. J. Chin, T. S. Ong, and A. B. J. Teoh, "Integrated biometrics template protection technique based on fingerprint and palmprint feature-level fusion," Information Fusion, vol. 18, pp. 161-174, 2014.

[15] J. Jo, S. J. Lee, and K. R. Park, "Detecting driver drowsiness using feature-level fusion and user-specific classification,” Expert Systems with Applications, vol. 41, pp. 1139-1152, 2014.

[16] S. Z. Li and W. Wu, "Feature learning based on SAE-PCA network for human gesture recognition in RGBD images," Neurocomputing, vol. 151, pp. 565-573, 2015.

[17] Y.-L. Boureau, J. Ponce, and Y. Lecun, "A theoretical analysis of feature pooling in visual recognition," ICML, 2010.

[18] X. B. Geng, T. Y. Liu, T. Qin, and H. Li, "Feature selection for ranking," SIGIR, Vol. 7, Month 1-2, 2007.

[19] M. S. Mohamad, S. D. S. M. Yatim, and M. R. Othman, "Feature selection method using genetic algorithm for the classification of Small and high dimension data," in Proc. First International Symposium on Information and Communications Technologies, October 7-8, 2004.

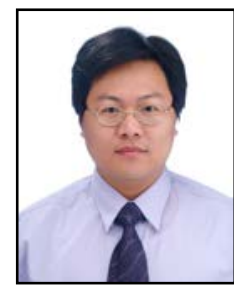

Ming-Chi Wu received the B.S. degree from Institute of Medicine, Chung Shan Medical University, Taiwan. He is currently the chief of division of neuroimaging, Department of Medical Imaging, Chung Shan Medical University Hospital, Taiwan. His research interests include medical image interpretation and diagnostic radiology.

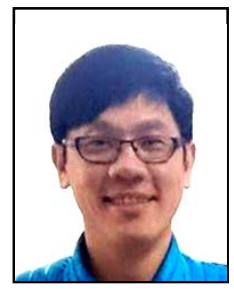

Chiun-Li Chin received the B.S. degree in computer science and information engineering and the M.S. degree in electrical and control engineering from Chung Hua University, Hsin Chu, Taiwan, and the Ph.D. degree in electrical and control engineering from National Chiao Tung University, Hsin Chu, Taiwan. From 2014 to 2015, he was a chair of department of medical informatics in Chung Shan Medical University. He is currently an associate professor of Department of Medical Informatics in Chung Shan Medical University. His research interests include medical image processing, computer vision, pattern recognition, and Healthcare software design.

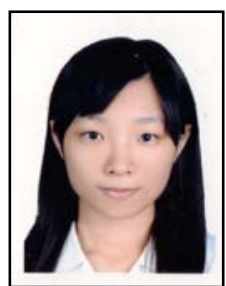

Wen-Chi Chin is currently studying in Chung Shan Medical University and doing her B.S. degree. Her research interests include medical image processing and pattern recognition. She has even been invited to U.K. to publish her paper about medical image processing. She also took part in many competitions in informatics and got good grades.

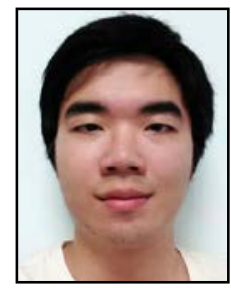

Jian-Shiun Wu is currently studying in Chung Shan Medical University, Taichung, Taiwan and doing his B.S. degree. His research interests include medical image processing and pattern recognition. He took part in many competitions in informatics and got good grades, and even been invited to London to do oral presentation in 2016.

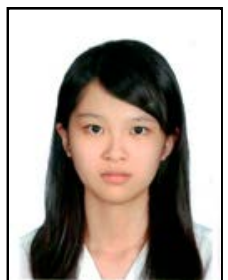

Wan-Jou Li is currently studying in Chung Shan Medical University, Taichung, Taiwan and doing her B.S. degree. Her research interests include medical image processing and pattern recognition. She took part in many competitions in informatics and got good grades, and even been invited to London to do oral presentation in 2016

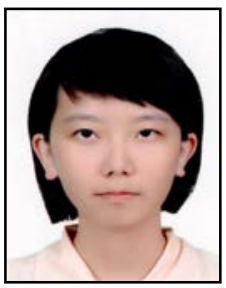

Ting-Yu Liu is currently studying in Chung Shan Medical University, Taichung, Taiwan and doing her B.S. degree. Her research interests include medical image processing and pattern recognition She took part in many competitions in informatics and got good grades, and even been invited to London to do oral presentation in 2016. 